\title{
Orbital simulations on the deflection of Near Earth Objects by directed energy
}

\author{
Qicheng Zhang*a, Kevin J. Walsh ${ }^{\mathrm{b}}$, Carl Melis ${ }^{\mathrm{c}}$, Gary B. Hughes ${ }^{\mathrm{d}}$, Philip M. Lubin ${ }^{\mathrm{a}}$ \\ * qicheng@cometary.org \\ ${ }^{a}$ Dept. of Physics, Univ. of California, Santa Barbara, CA USA 93106-9530; \\ bSouthwest Research Institute, Boulder, CO USA 80302; \\ ${ }^{\mathrm{c}}$ Center for Astrophysics and Space Sciences, Univ. of California, San Diego, CA USA 92093-0424; \\ ${ }^{d}$ Statistics Dept., California Polytechnic State Univ., San Luis Obispo, CA USA 93407-0405
}

\begin{abstract}
Laser ablation of a Near Earth Object (NEO) on a collision course with Earth produces a cloud of ejecta which exerts a thrust on the NEO, deflecting it from its original trajectory. The DE-STAR system provides thrust by illuminating an Earth-targeting asteroid or comet from afar with a stand-off system consisting of a large phased-array laser in Earth orbit. A much smaller version of the same system called DE-STARLITE travels alongside the target, operating in a stand-on mode, slowly deflecting it over a long period. Such a stand-on system would also permit directing the thrust in any desired direction through careful positioning of the laser relative to the target. This paper presents orbital simulations comparing the effectiveness of both systems across a range of laser and NEO parameters. Simulated parameters include magnitude, duration and, for the stand-on system, direction of the thrust, as well as the type, size and orbital characteristics of the target NEO. These simulations indicate that deflection distance is, in general, proportional to the magnitude of thrust, proportional to the square of the laser on time, and inversely proportional to the mass. Furthermore, deflection distance shows strong dependence on thrust direction with optimal direction varying with the object's orbital eccentricity. As one example, consider a $325 \mathrm{~m}$ asteroid in an orbit of eccentricity e=0.2: given 15 years of warning, a force of just $2 \mathrm{~N}$ from a stand-on DE-STARLITE system is sufficient to deflect the asteroid by 2 Earth radii. Numerous scenarios are discussed as is a practical implementation of such a system consistent with current launch vehicle capabilities.
\end{abstract}

Keywords: asteroid deflection, comet deflection, directed energy, laser ablation, Near Earth Object, orbital simulation

\section{INTRODUCTION}

A wide array of concepts for the deflection of threatening Near Earth Objects (NEO) have been proposed. Several detailed surveys of threat mitigation strategies are available such as [1], [2], [3], [4] and [5]. These strategies fall into several categories including

1. Kinetic impactors, with or without explosive charges. An expendable spacecraft would be sent to intercept the threatening object. Direct impact would modify the object's orbit through momentum transfer. Enhanced momentum transfer can occur using an explosive charge, such as a nuclear weapon, e.g. [6], [7], [8], [9].

2. Gradual orbit deflection by surface albedo alteration. The albedo of an object could be changed using paint ([10]), mirrors ([11]), sails ([12]), etc. As the albedo is altered, a change in the object's Yarkovsky thermal drag would gradually shift the object's orbit.

3. Direct motive force, such as by mounting a thruster directly to the object. Thrusters could include chemical propellants, solar or nuclear powered electric drives, or ion engines [13]. 
4. Indirect orbit alteration, such as gravity tractors. A spacecraft with sufficient mass would be positioned near the object, and maintain a fixed station with respect to the object using on-board propulsion. Gravitational attraction would tug the object toward the spacecraft, and gradually modify the object's orbit [14].

5. Expulsion of surface material such as by robotic mining. A robot on the surface of an asteroid would repeatedly eject material from the asteroid. The reaction force when material is ejected affects the object's trajectory [16].

6. Vaporization of surface material. Like robotic mining, vaporization on the surface of an object continually ejects the vaporized material, creating a reactionary force that pushes the object into a new path. Vaporization can be accomplished by solar concentrators ([17]) or lasers ([18]) deployed on spacecraft stationed near the asteroid. One study envisioned a single large reflector mounted on a spacecraft traveling alongside an asteroid [19]. The idea was expanded to a formation of spacecraft orbiting in the vicinity of the asteroid, each equipped with a smaller concentrator assembly capable of focusing solar power onto an asteroid at distances near $\sim 1 \mathrm{~km}$ [20]. Efficiency of a laser system for surface ablation can be enhanced using an array of phase-locked lasers ([21]), allowing more photonic flux to be delivered to the asteroid and at greater distances. Envisioning ever larger arrays of phase-locked lasers allows contemplation of stand-off systems that could deliver sufficient flux to the surface of a distant asteroid from Earth orbit [22].

Simulations were developed in order to measure the effectiveness of deflection of a threat by laser ablation as proposed in [21] and [22]. Both stand-off and stand-on missions are discussed. The much larger stand-off system is called DESTAR for Directed Energy System for Targeting of Asteroids and exploRation and consists of a laser which remains in Earth orbit ablating the target from afar. A much smaller ("lite") stand-on system called DE-STARLITE involves a laser being physically delivered to the target. The laser technology is described in much greater detail in [21], [22] and [27]. The effects of asteroid rotation are discussed in [25] and the optical modeling is discussed in [26] and [28].

Emphasis is placed on the more practical stand-on system which can be built more rapidly and inexpensively as a nearterm solution due to its reduced scale. However, the full stand-off system is still considered for its ability to target threats like long-period comets in orbits unreachable by modes of propulsion.

\subsection{Laser ablation of an asteroid's surface}

In a stand-on DE-STARLITE system, photovoltaic arrays are used to obtain $\sim 100 \mathrm{~kW}$ electrical power. The objective of the laser directed energy system is to project a large enough flux onto the surface of the asteroid to heat the surface to a temperature that exceeds the vaporization point of constituent materials, typically around 2000-3000 K, or a flux in excess of $10^{7} \mathrm{~W} / \mathrm{m}^{2}$. A reactionary thrust due to mass ejection will divert the asteroid's trajectory.

To produce sufficient flux, the system must have both adequate beam convergence and sufficient power. Optical aperture size, pointing control and jitter, and efficacy of adaptive optics techniques are several critical factors that affect beam convergence. The optical power output of DE-STARLITE can be varied depending on the target size and warning time and is typically between 1 and $1000 \mathrm{~kW}$. The current laser "wallplug efficiency" baselines for DE-STAR and DESTARLITE are nearing 50\%. Even higher efficiency allows for more thrust on the target for a given electrical input as well as for smaller radiators and hence lower mission mass. From a distance of $10 \mathrm{~km}$, DE-STARLITE is capable of projecting a spot size on the asteroid of $10 \mathrm{~cm}$, providing enough flux to vaporize rock [21].

Evaporation at the spot produces a vaporization plume thrust that can be used to change the asteroid's orbit and effectively deflect asteroids from colliding with Earth. In [22], [25] and [27], simulations are performed with the high temperature materials expected in rocky target that require the highest flux and the low temperature volatiles in comets that can also be deflected with much less flux.

DE-STARLITE can be designed for a variety of power levels, depending on the target's needs, which range typically from 1-1000 kW (optical) with thrust of approximately $100 \mu \mathrm{N} / \mathrm{W}$. Smaller and larger asteroid or comets with less or greater warning time can used DE-STARLITE systems that are simply scaled. This paper uses estimated thrust levels expected from simulations and measurements for DE-STAR and DE-STARLITE as inputs for orbital simulations of NEO deflection scenarios. 


\section{ORBITAL SIMULATIONS}

The simulation considers the 3-body system consisting of the Sun, Earth and asteroid with all three modeled as Newtonian gravitational point sources. The Moon is not considered as a separate body, but its mass is combined with that of the Earth. This combined "Earth-Moon point mass" is denoted here simply as the Earth. The acceleration of the asteroid as numerically integrated is

$$
\mathbf{a}=\mathbf{a}_{\mathrm{g}}+\mathbf{a}_{\mathbf{1}}
$$

where the first component $\mathbf{a}_{\mathbf{g}}$ is the net gravitational acceleration from the Sun and Earth. The second component $\mathbf{a}_{\mathbf{l}} \equiv a_{l} \hat{\mathbf{a}}_{\mathbf{1}}$ is the acceleration from the laser's thrust $F=m a_{l}$ for an asteroid of mass $m$. For these simulations, the asteroid is assumed to be spherical with a uniform density of $\rho=2000 \mathrm{~kg} / \mathrm{m}^{3}$. The direction of thrust $\hat{\mathbf{a}}_{\mathbf{l}}$ varies depending on the mode by which the thrust is applied.

\subsection{Stand-on mode}

In the stand-on thrust case, the laser is maneuvered in close proximity to the target asteroid. It may therefore be positioned off any side of the asteroid so the generated thrust may be oriented in any direction. The model considers the special case where the direction of thrust $\hat{\mathbf{a}}_{\mathbf{1}}$ is fixed relative to the direction of the asteroid's velocity $\hat{\mathbf{v}}$ and that of its orbital momentum $\hat{\mathbf{I}}$. $\hat{\mathbf{a}}_{\mathbf{I}}$ may then be specified in the frame defined by these directions by an azimuth angle $\alpha$ and elevation angle $\beta$ :

$$
\hat{\mathbf{a}_{\mathbf{l}}}=\hat{\mathbf{v}} \cos \alpha \cos \beta+(\hat{\mathbf{l}} \times \hat{\mathbf{v}}) \sin \alpha \cos \beta+\hat{\mathbf{l}} \sin \beta
$$

with $\hat{\mathbf{l}} \equiv \hat{\mathbf{r}} \times \hat{\mathbf{v}}$.

Because the laser is near the target asteroid, the spot size of the laser on the asteroid is smaller than the asteroid itself, so the magnitude of thrust on the asteroid is simply $F=F_{0}=m a_{l}$.

\subsection{Stand-off mode}

In the stand-off thrust case, the laser is a satellite in orbit around the Earth, both of which are considered to be at a common position $\mathbf{r}_{\mathbf{e}}$. From a distance, the laser ablates material off the Earth-facing side of the asteroid at position $\mathbf{r}$. Therefore, the thrust on the asteroid must be in the direction of its geocentric position vector:

$$
\hat{\mathbf{a}_{1}}=\frac{\mathbf{r}-\mathbf{r}_{\mathrm{e}}}{\left\|\mathbf{r}-\mathbf{r}_{\mathrm{e}}\right\|}
$$

Because the laser operates at a large distance from the target, the laser beam must diverge due to diffraction effects. At a distance $\Delta \equiv\left\|\mathbf{r}-\mathbf{r}_{\mathbf{e}}\right\|$, a phased-array laser of diameter $d$ produces a spot roughly of diameter

$$
D_{s}=\frac{2 \lambda}{d} \Delta
$$

Approximating the spot illumination as uniform and thrust as proportional to incident power, the thrust on an asteroid of diameter $D$ is

$$
F=F_{0} \times\left\{\begin{array}{r}
1 \text { if } D_{s} \leq D \\
\left(D / D_{s}\right)^{2} \text { if } D_{s}>D
\end{array}\right.
$$

where $F_{0}$ is the thrust produced by ablation with the full power $P$ of the laser. 
Note, however, that for laser ablation and thus significant thrust generation to occur, $D_{s}$ must be smaller than some power and target-dependent $D_{\text {crit }}$. When $D_{s}>D_{\text {crit }}$, there is insufficient flux density to raise the temperature on the target to its vaporization temperature $T_{c r i t}$ and thus activate the ablation process. This temperature is only reached when $\Delta$ is below some critical distance $\Delta_{\text {crit }}$. To estimate $\Delta_{\text {crit }}$, the target is approximated as a perfect blackbody with radiation being the only mode of transport for thermal energy. Then,

$$
\Delta_{\text {crit }}=\frac{d}{\lambda} \sqrt{\frac{P}{\pi \sigma T_{\text {crit }}^{4}}}
$$

In addition, to prevent cancellation of thrust over time, the laser should only be activated for a consistent sign of the quantity

$$
\xi=\left(\mathbf{r}-\mathbf{r}_{\mathbf{e}}\right) \cdot \mathbf{v}
$$

where $\mathbf{V}$ is the heliocentric velocity of the asteroid. The sign of $\xi$ defines whether the Earth is ahead $(\xi<0)$ or behind $(\xi>0)$ the asteroid in its orbit which determines whether thrust from the laser advances or delays the motion of the asteroid respectively.

\subsection{Initial conditions}

The orbit of a target asteroid is characterized by three parameters: semi-major axis $(a)$, eccentricity $(e)$ and inclination to the ecliptic (i). Its intersection with Earth in space and time constrains the remaining three degrees of freedom. These simulations use an intersection at Earth's aphelion. However, due to the near circular shape of Earth's orbit, simulation results are nearly identical for other points of intersection.

Time of impact is designated to be $t=T$ for some specified $T>0$ and is defined to be when the Earth and the asteroid occupy the same position $\mathbf{r}(T)$. As the Keplerian orbital elements are only relevant for two-body systems, the gravity of the Earth is temporarily neglected to fit a Keplerian orbit with the chosen orbital elements through the point of impact. With this two-body orbit and the two-body orbit of the Earth, $\mathbf{r}(T-\delta t)$ of the asteroid and $\mathbf{r}_{\mathbf{e}}(T-\delta t)$ of the Earth may be computed for a small $\delta t>0$. A $\mathbf{v}(T-\delta t)$ may also be computed from the two-body solution of the asteroid adjusted in magnitude with by potential well of the Earth at $t=T-\delta t$. Finally, the initial conditions of the asteroid $(\mathbf{r}(0), \mathbf{v}(0))$, and those of the Earth $\left(\mathbf{r}_{\mathbf{e}}(0), \mathbf{v}_{\mathbf{e}}(0)\right)$ may be computed by numerical integration with a time-reversed system.

\section{DEFLECTION SIMULATION RESULTS}

Deflection of asteroids using both stand-off thrust, provided by a DE-STAR system, and stand-on thrust, provided by a DE-STARLITE system, was considered for a range of asteroid sizes and orbits. Simulations were performed with a standard solar system N-body integrator package, SyMBA [30]. The standard integrator used by SyMBA is the mixed variable symplectic mapping (MVS) which recursively divides the time-step for two bodies suffering close approaches with each other.

The measure of the effectiveness of a given setup with a given target asteroid is the miss distance of the asteroid to the Earth. To compute the miss distance, integration is performed with SyMBA from $t=0$ through $t=T$. However, in addition to increasing miss distance, the thrust may also advance or delay the time of closest approach. Therefore, if $\Delta=\left\|\mathbf{r}-\mathbf{r}_{\mathrm{e}}\right\|$ continues to decrease at $t=T$, integration is continued until a minimum value of $\Delta$ is reached. This process produces a discrete set of times $t_{i}$ and their respective Earth-asteroid distances $\Delta_{i}$. Beginning at the very last step and moving in reverse, the first local minimum in $\Delta_{i}$ is found and labeled $\Delta_{k}$. To refine this distance, the state 
vectors $\mathbf{r}\left(t_{k-1}\right), \mathbf{v}\left(t_{k-1}\right)$ and $\mathbf{r}_{\mathbf{e}}\left(t_{k-1}\right), \mathbf{v}_{\mathbf{e}}\left(t_{k-1}\right)$ from the previous step at $t_{k-1}$ are used as the new initial state vectors for the asteroid and Earth respectively. Integration is then performed with the classical $4^{\text {th }}$ order Runge-Kutta method (RK4) with the same acceleration functions as used with SyMBA but with a smaller time-step, continuing through the point of minimum $\Delta$. The step with the smallest $\Delta=\Delta_{\min }$ is taken as the point of closest approach with $\Delta_{\min }$ as the miss distance.

The asteroid 99942 Apophis is a well-known case of a Potentially Hazardous Object. It is a relatively large Atens group asteroid with a diameter of approximately $325 \mathrm{~m}$ with an orbit of semi-major axis $a=0.92$ au, eccentricity $e=0.19$ and inclination $i=3.3^{\circ}$. Such an asteroid is considered here as the canonical "large" asteroid.

\subsection{Stand-on results}

For a stand-on mission, it is conceivable to achieve a thrust of up to $F=100 \mathrm{~N}$ with a $\sim 1 \mathrm{MW}$ laser. Such a thrust may deflect the $325 \mathrm{~m}$ asteroid to a miss distance of 2 Earth radii in as little as 2.5 years with thrust in the $\alpha=\beta=0^{\circ}$ direction which appears to be near the optimal direction for a laser active over several years. With a decade of laser activity, deflection to 2 radii is possible with less than $7 \mathrm{~N}$ thrust. Given 15 years of ablation, $2.5 \mathrm{~N}$ thrust is sufficient. In each case, besides a gravitational deviation by Earth at small thrust, miss distance grows roughly quadratically with increased time and linearly with increased thrust as seen in Figure 1.
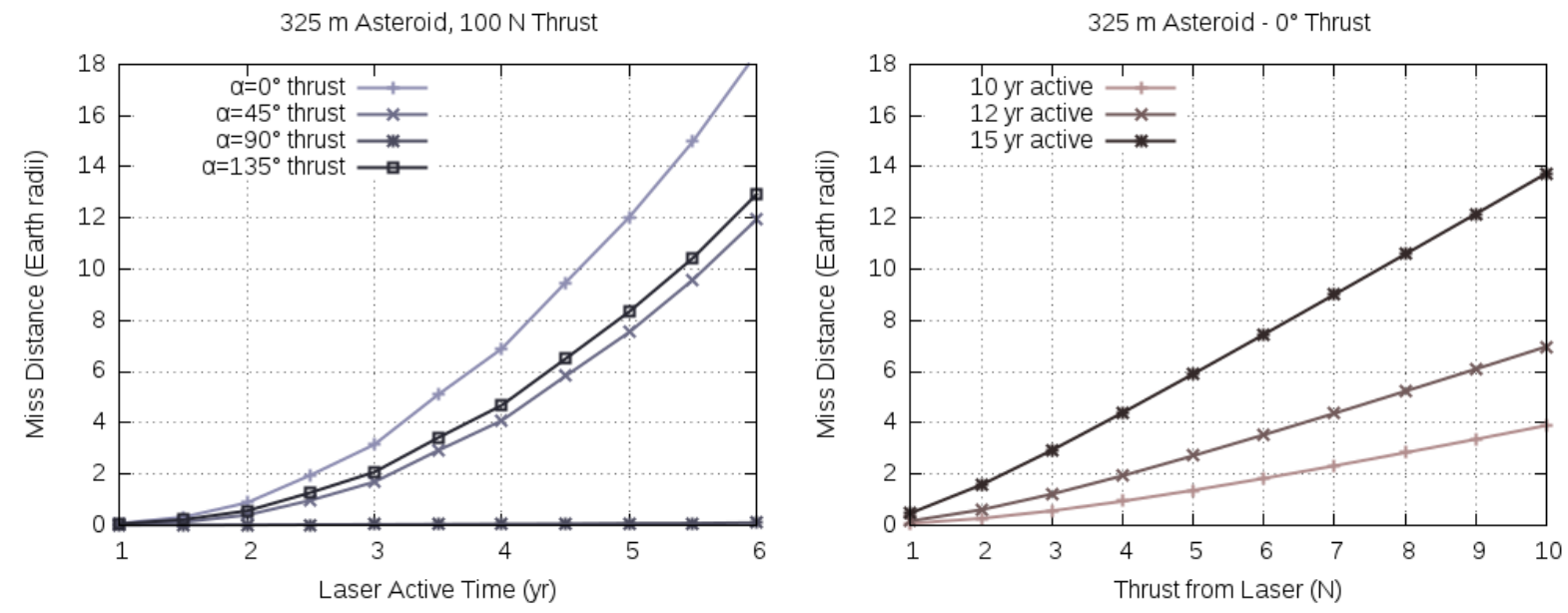

Figure 1. For $100 \mathrm{~N}$ applied to a $325 \mathrm{~m}$ asteroid (left), deflection distance increases quadratically with increasing time of laser activity. Thrust directed parallel to the asteroid's velocity vector is more effective than thrust directed $45^{\circ}, 90^{\circ}$ and $135^{\circ}$ from the velocity vector in the plane of the asteroid's orbit. Significantly less thrust of $<10 \mathrm{~N}$ in the parallel to velocity direction is needed to deflect the asteroid by 2 Earth radii if the available time for laser activity is increased to 10-15 years (right). Deflection distance varies approximately linearly with thrust.

For small deflections of under 100 Earth radii, miss distance scales roughly linearly with $a_{l}$, so scales inversely with mass and the cube of diameter as shown in Figure 2. A large $100 \mathrm{~N}$ stand-on mission can deflect a $500 \mathrm{~m}$ asteroid in an Apophis-like orbit by 2 radii in under 5 years. Alternatively, a $350 \mathrm{~m}$ asteroid can be deflected in 3 years or a $170 \mathrm{~m}$ asteroid in less than 1 year. 
5 yr On Target $-0^{\circ}$ Thrust

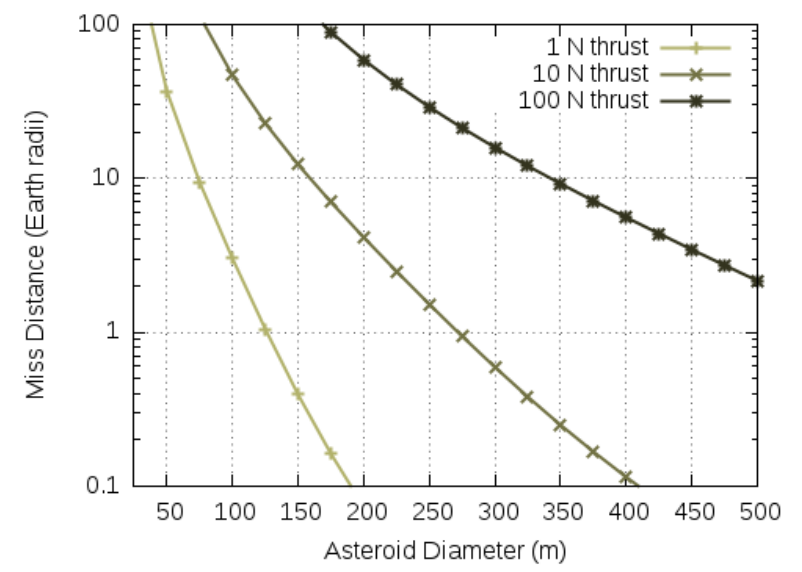

100 N Thrust $-0^{\circ}$ Thrust

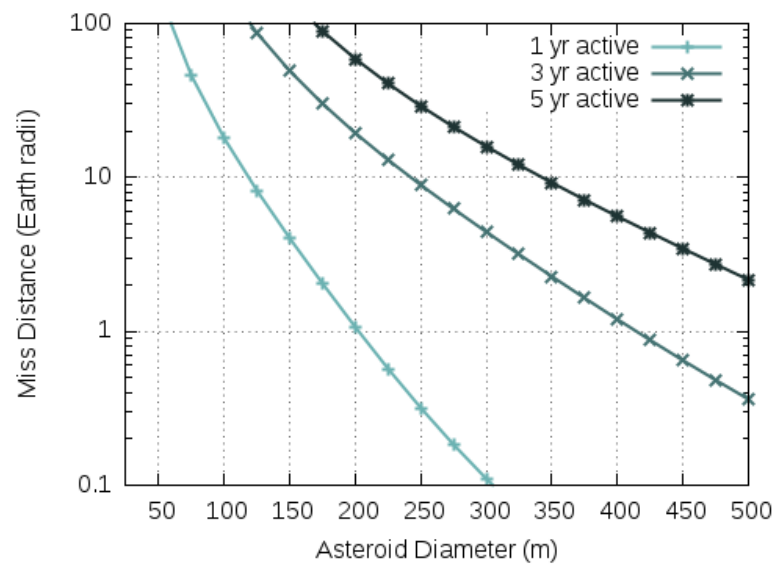

Figure 2. Miss distance scales inversely with the mass of the target asteroid and the cube of its diameter. With 5 years of deflection (left), a $500 \mathrm{~m}$ asteroid may be deflected by 2 Earth radii with $100 \mathrm{~N}$ thrust, a $230 \mathrm{~m}$ asteroid with $10 \mathrm{~N}$ thrust or a $100 \mathrm{~m}$ asteroid with $1 \mathrm{~N}$ thrust. A large $100 \mathrm{~N}$ stand-on system (right) can also deflect a $350 \mathrm{~m}$ asteroid in 3 years or a 170 $\mathrm{m}$ asteroid in 1 year by the same amount.

Smaller $80 \mathrm{~m}$ asteroids - roughly the size of the 1908 Tunguska impactor - can be deflected with $100 \mathrm{~N}$ thrust in less than 6 months. For targets of this size or smaller, the laser might only be active for a fraction of the laser's orbit. As a result, the effect of the $0^{\circ}$ thrust in delaying the asteroid's arrival at the impact point is now less effective due to the opposing effect of $0^{\circ}$ thrust acting to speed up the asteroid locally in its orbit.

The simulations show, in Figure 3, that as the time on target of the laser decreases, the optimal values of angles $\alpha$ and $\beta$ both shift from $0^{\circ}$ towards $90^{\circ}$. However, while the optimal $\alpha$ begins to shift as laser active time approaches 0.9 years (approximately one orbit of the asteroid), the optimal $\beta$ remains nearly fixed at $0^{\circ}$ until laser active time drops below 6 months. This result is consistent with the notion that shifting the path of the asteroid within the plane of its orbit (with $\alpha=90^{\circ}$ ) requires significantly less total impulse than shifting the orbital plane itself (with $\beta=90^{\circ}$ ). 

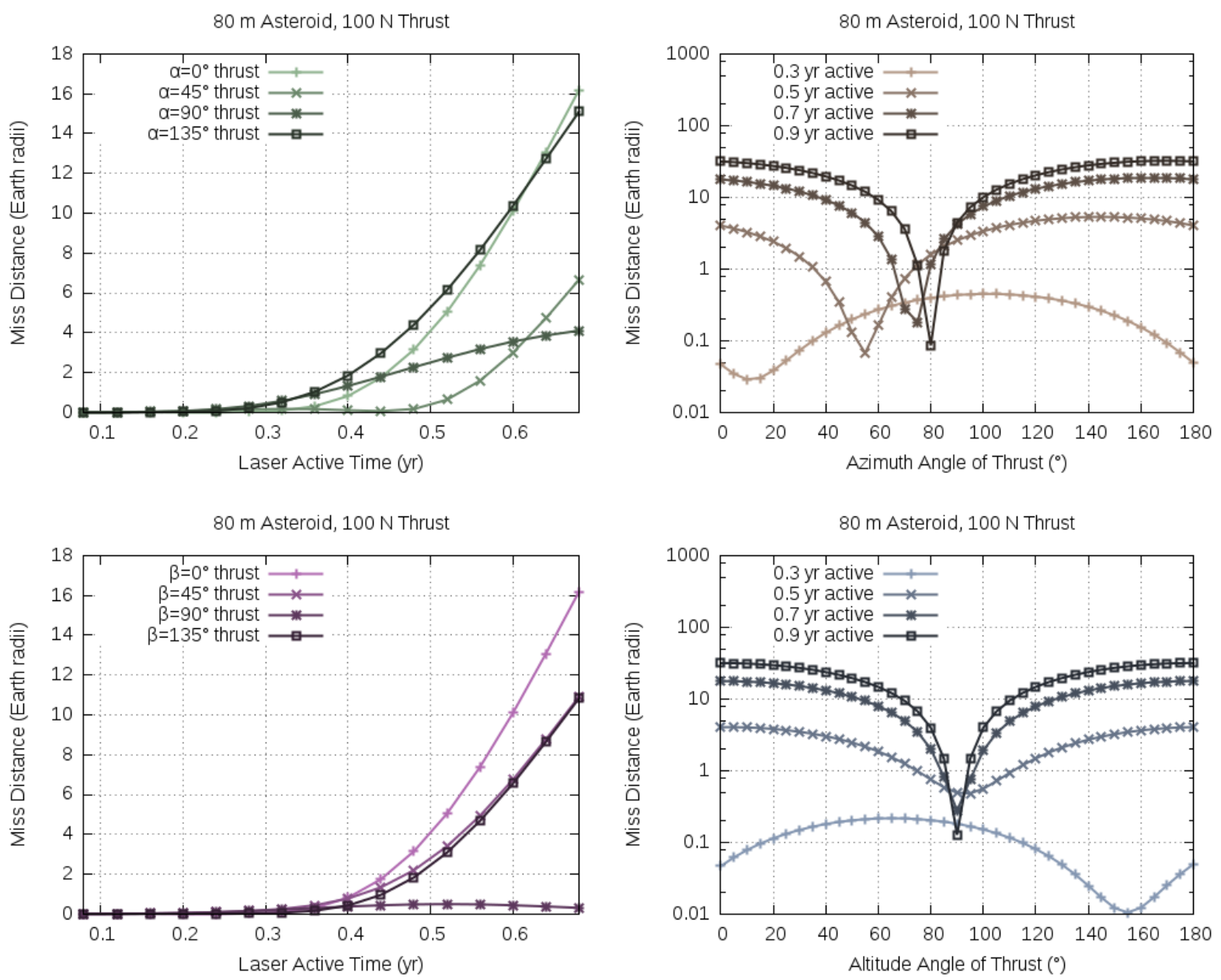

Figure 3. A $100 \mathrm{~N}$ thrust laser acts on an $80 \mathrm{~m}$ asteroid beginning less than one orbit before the asteroid's encounter with the Earth. As the time on target of the laser decreases to less than one orbital period, the optimal direction for thrust deviates away from that of the asteroid's velocity and towards being orthogonal to the velocity. The effect is already pronounced at one period ( 0.9 years) in the azimuth $(\alpha)$ direction with a shift of $10^{\circ}$ (upper) while a similar shift in the altitude $(\beta)$ direction (lower) is only attained for a laser active over half of a period ( 0.45 years).

In addition, the orbit of the target asteroid also affects the effectiveness of thrust in deflecting the asteroid. To measure these effects, a $325 \mathrm{~m}$ asteroid in an Apophis-like orbit is taken with $e$ and $i$ varied independently in ranges typical of known Near Earth Asteroids. Simulations of asteroids in these orbits suggest that for a given amount of thrust, deflection distance grows as the orbit of the asteroid becomes more different from the Earth's - that in general, larger $e$ and larger $i$ correspond to increased deflection up to a point, beyond which there is little change as seen in Figure 4.

Note, however, that this result does not imply that asteroids with orbits very different from the Earth are easier to deflect with a stand-on mission. In general, orbits with large $e$ and $i$ require a large $\Delta v$ to reach from Earth. Therefore, the total mass and therefore total power of the laser to be delivered to the asteroid is lower for a given launch cost. The mild gains in deflection per thrust from a highly eccentric and inclined orbit are generally offset by this much more significant reduction in thrust. 

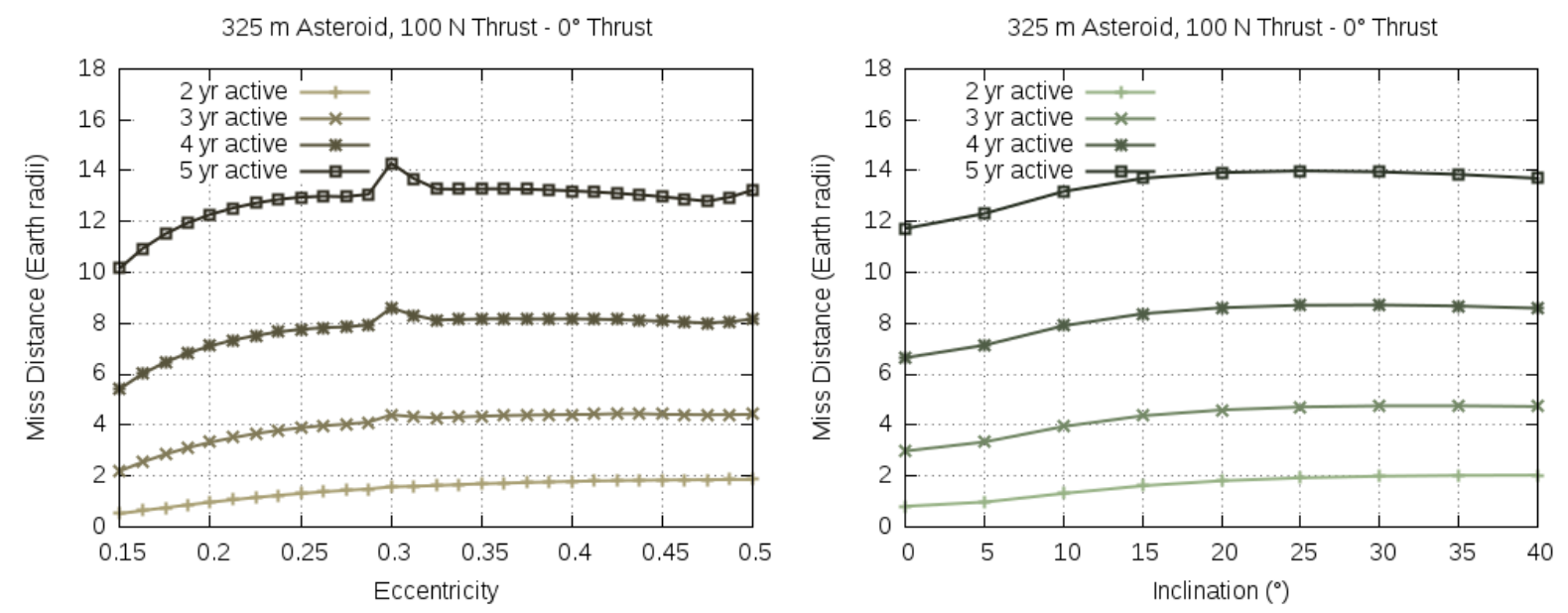

Figure 4. Revisiting the case of $100 \mathrm{~N}$ applied to a $325 \mathrm{~m}$ asteroid: the orbit of Apophis was taken and its eccentricity and inclination was independently varied and the deflection distance results were compared. The miss distance of the asteroid is generally larger for an orbit with higher eccentricity (left) and greater inclination (right) up to a point beyond which deflection distance flattens out. Note the obvious bumps in deflection for an orbit of $e=0.3$ with all four of the tested durations of laser activity. The cause for these bumps has not been determined.

\subsection{Stand-off results}

In contrast to a stand-on mission where any laser activity is preceded by a potentially lengthy transit period, a stand-off system may be used as soon as an asteroid is identified as a threat. Being limited by $\Delta_{\text {crit }}$, stand-on systems are generally limited to operation over very short timescales on the order of a few days or weeks unless the phased array is at least several kilometers in diameter which is difficult to build. Systems of such scale are necessary to deflect larger asteroids like Apophis.

However, arrays as small as $d=1 \mathrm{~km}$ may still be effective against Tunguska-class impactors. Such a $1 \mathrm{~km}$ array could intercept $1.4 \mathrm{GW}$ of solar power. Operating at $50 \%$ efficiency, it would produce $P=0.7 \mathrm{GW}$ and $F=70 \mathrm{kN}$ $(100 \mu \mathrm{N} / \mathrm{W})$ and may deflect an $80 \mathrm{~m}$ asteroid in an Apophis-like orbit with a vaporization point of $T_{\text {crit }}=2500 \mathrm{~K}$ by a distance of 0.3 Earth radii over the course of 4 weeks. While generally insufficient to prevent an impact, a deflection of this magnitude is more than sufficient to relocate the impact ellipse to a favorable site.

Notice in Figure 5 that the distance of deflection is strongly dependent on the thrust. Improving laser efficiency to $70 \%$ (far beyond current capability) with $1 \mathrm{GW}$ power and $100 \mathrm{kN}$ thrust significantly increases the deflection distance to 2 Earth radii which is sufficient to prevent an impact completely given a well-determined orbit a month early. Activating the laser earlier than about 1 month before impact yields no additional deflection. 

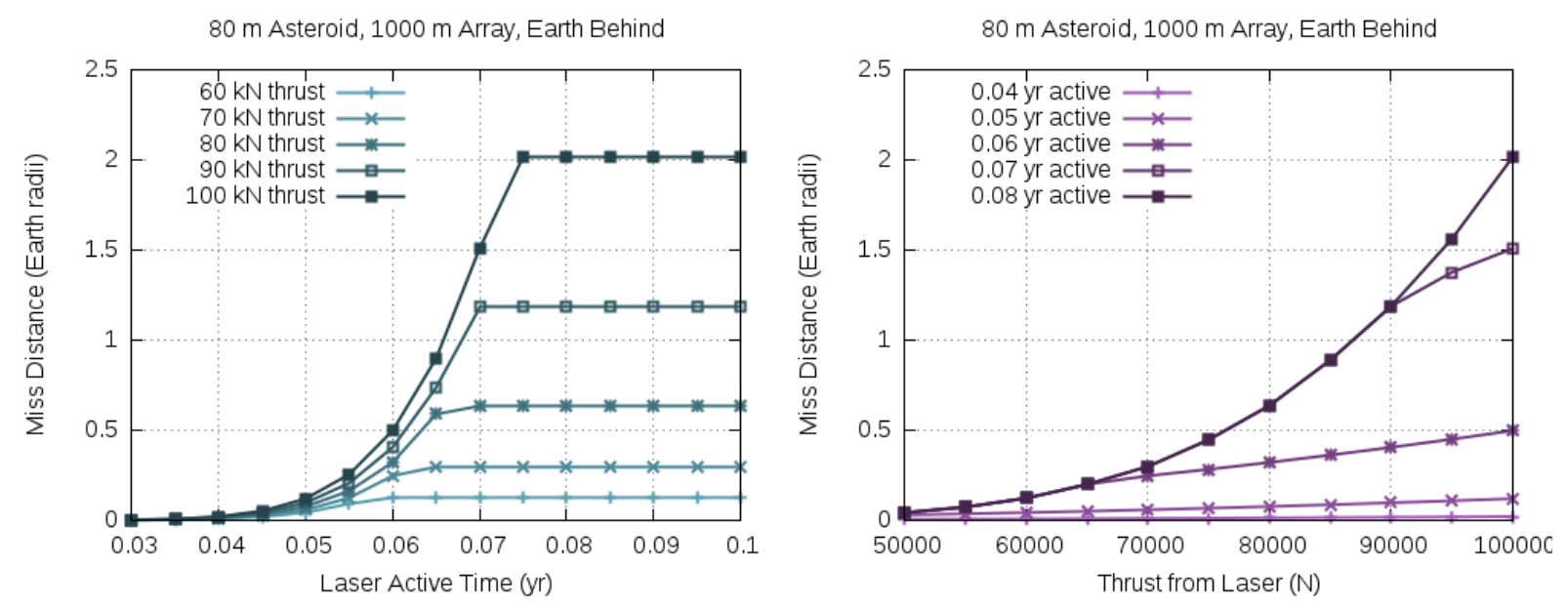

Figure 5. A $1 \mathrm{~km}$ phased-array laser deflects an $80 \mathrm{~m}$ Tunguska-class asteroid from Earth orbit. Deflection distance grows like the stand-on case with laser active time to a certain point before flattening out as activating the laser before the asteroid approaches to within $\Delta_{\text {crit }}$ yields no additional effect on deflection distance (left). In addition, deflection distance grows faster than linearly with increased thrust given the confirmation of a threat before the asteroid reaches $\Delta_{\text {crit }}$ since increased power increases $\Delta_{\text {crit }}$ and therefore increases the time in which ablation occurs (right). Otherwise, linear growth with thrust is observed for cases when the flux is sufficient for ablation to occur over the full period as was observed in stand-on mode. At large thrust and thus large power, $\Delta_{\text {crit }}$ is larger and so the period over which ablation occurs is longer, eventually covering the entire duration of laser activity. As a result, there is a transition from greater-than-linear growth, a characteristic of varying laser time, to linear growth, a characteristic of constant time, in deflection with thrust.

An efficiency of $50 \%$ corresponds to a thrust of $70 \mathrm{kN}$ which is generally insufficient to deflect the asteroid from an impact with the Earth. The deflection of 0.3 Earth radii, however, may be sufficient to move the site of impact away from a populated area. An even larger $1.2 \mathrm{~km}$ array (not shown) is needed to deflect the asteroid by a safe 2 radii.

Unlike the case of a stand-on mission which must be delivered to its target, a stand-off system in Earth orbit can target and provide thrust to objects approaching the Earth in any direction. As a result, objects like long period or dynamically new comets with highly eccentric and often very inclined orbits can be targets despite being physically inaccessible by the extreme $\Delta v$ between the Earth's orbit and that of the target.

Furthermore, comets are being heated by solar radiation to a temperature where its ices are already vaporizing. While this behavior increases the difficulty of predicting a comet's trajectory and thus determining whether the comet is a threat, it also extends the range of ablation to the entire zone around the Sun in which a comet will display cometary behavior, a condition that must usually be satisfied for the comet to be sufficiently bright for discovery. The additional energy from the laser beam increases the thrust from the Sun already acting on the comet and further perturbs its trajectory, deflecting it from an otherwise collisional trajectory.

Figure 6 shows how flux declines with distance for $500 \mathrm{~m}, 1 \mathrm{~km}$ and $2 \mathrm{~km}$ arrays compared with the flux from the Sun. The flux needed to vaporize typical basaltic rocks and water ice are also included to show the maximum range $-\Delta_{\text {crit }}-$ at which each source can ablate the surface an asteroid and water ice on a comet. The $\Delta_{\text {crit }}$ for water ice from the Sun alone extends to $2 \mathrm{au}$. Any comet passing within that distance of the Sun - which is necessary for the comet to be a threat to Earth - already receives sufficient flux from the Sun to vaporize water ice, hence the cometary behavior. In addition, most comets - especially long period and dynamically new comets - contain a significant fraction of other volatiles that vaporize at even lower fluxes and so have even larger $\Delta_{c r i t}$. For these simplified orbital simulations, the comet is assumed to have been discovered while active and thus receives sufficient flux from the Sun alone for vaporization to occur. 


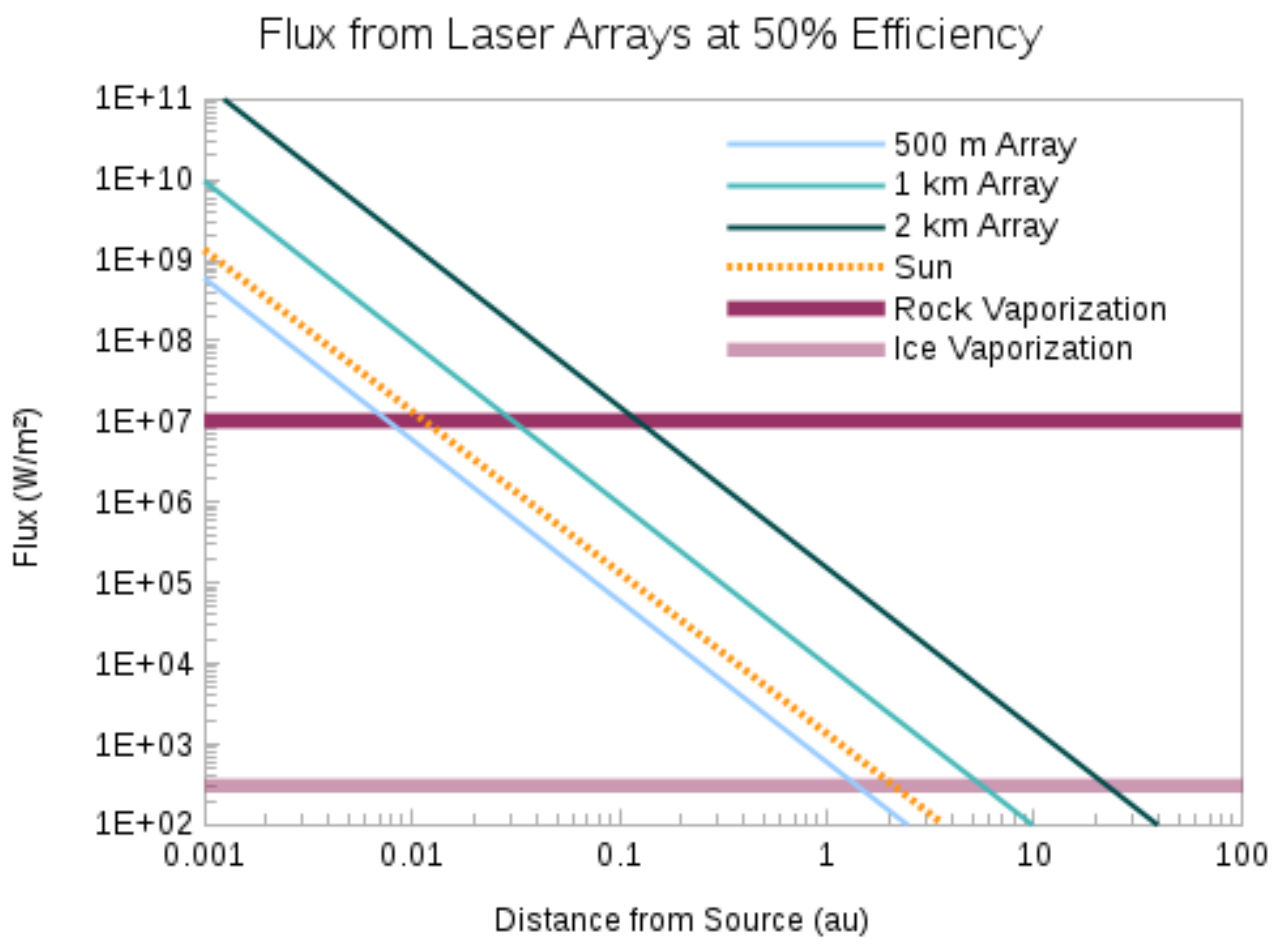

Figure 6. Each material has a vaporization flux above which the material will vaporize. Rocky material found in most asteroids vaporize when the flux is above about $10^{7} \mathrm{~W} / \mathrm{m}^{2}$ (dark purple bar) while water ice, as found on comets, vaporizes at just $300 \mathrm{~W} / \mathrm{m}^{2}$ (light purple bar). Other volatiles found on comets need even lower fluxes for vaporization. Laser flux (diagonal blue lines) falls off with the square of distance from the laser, so there is a distance limit $-\Delta_{\text {crit }}-$ beyond which the flux is too low to vaporize material.

Due to the significantly lower flux needed to vaporize water ice compared to rock, the $\Delta_{\text {crit }}$ for ablating ice off the surface of a comet is a 200 times larger than the $\Delta_{\text {crit }}$ needed to vaporize rock from an asteroid. In the case of comets, even the Sun (dotted orange line) has a profound effect as its $\Delta_{\text {crit }}$ for water ice stretches out to 2 au. Any comet passing within that distance from the Sun - necessary for the comet to be a threat to Earth - already receives sufficient flux from the Sun to vaporize water ice. Any additional flux, such as from a laser, will add to the thrust already generated by the Sun producing a deflection from the comet's natural trajectory.

Notice that a laser array of $\sim 600 \mathrm{~m}$ or larger will generate a higher flux than the Sun does at any point an equal distance away from both. A large $2 \mathrm{~km}$ array can extend the zone where water ice vaporizes to over $20 \mathrm{au}$, the orbit of Uranus.

Simulations were run assuming a simple spherically symmetric comet with density $600 \mathrm{~kg} / \mathrm{m}^{3}$ in an orbit with perihelion $q=0.8 \mathrm{au}$, eccentricity $e=0.98$ and inclination $i=130^{\circ}$. Thrust from the laser is assumed to be radial as with the asteroid cases and it similarly falls off with $1 / \Delta^{2}$ when the beam size exceeds the size of the comet. In addition, although the effect of heating from the Sun may be more significant than the effect on heating by the laser, the deviation from these solar non-gravitational effects is still small relative to the overall shape of the comet's trajectory and is thus ignored for these simulations which consider only the deviation caused by the laser from the natural trajectory. As with the asteroid cases, $100 \mu \mathrm{N} / \mathrm{W}$ is assumed. Figure 7 illustrates the orbital deflection of a $500 \mathrm{~m}$ comet by a $1 \mathrm{~km}$ array. Figure 8 shows that with the $1 \mathrm{~km}$ array, a $500 \mathrm{~m}$ comet may be deflected by 30 radii or a $2 \mathrm{~km}$ comet by 5 Earth radii given 2 years of warning. 


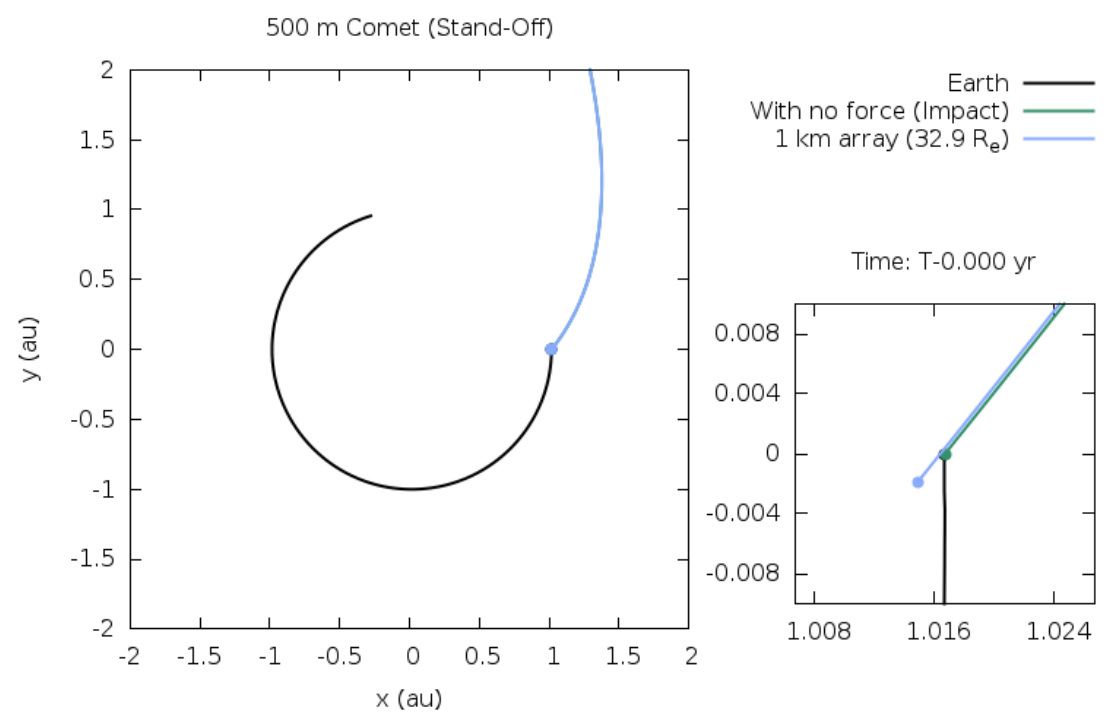

Figure 7. Long period comets passing through the inner solar system often pass perihelion (and thus the Earth) in under 2 years after discovery. Such short notice coupled with the highly eccentric and inclined orbits of many of these comets makes the delivery of a stand-on system to a threatening comet infeasible, making a stand-off system the only possibility for deflection with directed energy. In this figure, a $500 \mathrm{~m}$ comet with $e=0.98$ and $i=130^{\circ}$ (green) approaches from above and impacts the Earth (black) approaching from below in a near head-on collision. Activating a $1 \mathrm{~km}$ stand-off array 2 years in advance leads the deflected comet (light blue) to arrive at the Earth's orbit before the Earth, averting the impact.

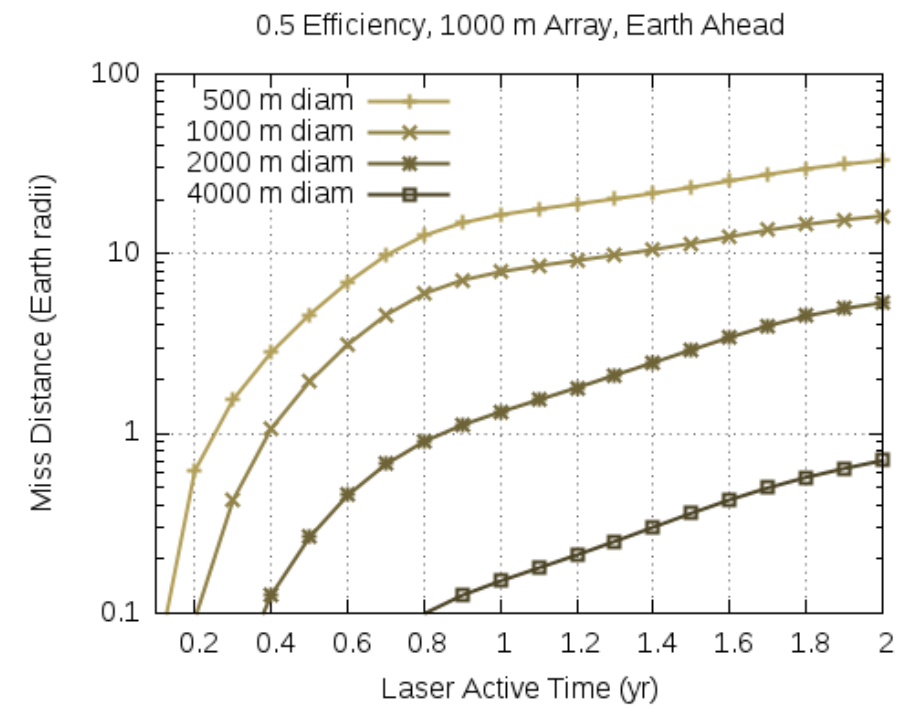

Figure 8 . A $1 \mathrm{~km}$ laser array at 50\% efficiency produces $70 \mathrm{kN}$ thrust which can deflect a comet as large as $2 \mathrm{~km}$ by 5 Earth radii given 2 years of warning. A more typical $500 \mathrm{~m}$ comet can be deflected by a much larger 30 radii, a distance generally large enough to overcome the anticipated uncertainties in the comet's computed trajectory.

The effectiveness drops rapidly as the arrays are scaled down. The minimum useful size of an array is about $400 \mathrm{~m}$. Such an array in Earth orbit operating at 50\% efficiency yields $F=11 \mathrm{kN}$. This $400 \mathrm{~m} / 11 \mathrm{kN}$ system can deflect a small $80 \mathrm{~m}$ comet by 2 Earth radii in one year or 5 radii in two. As shown in Figure 9, increasing the laser active time $T$ increases deflection distance roughly quadratically for small $T$. For larger $T$, the laser activity begins before the comet is sufficiently close to intercept the entire laser beam resulting in a deflection distance that increases less than 
quadratically. Improving laser efficiency and thus increasing the thrust exerted on the comet scales with deflection distance linearly just as with the stand-on cases.
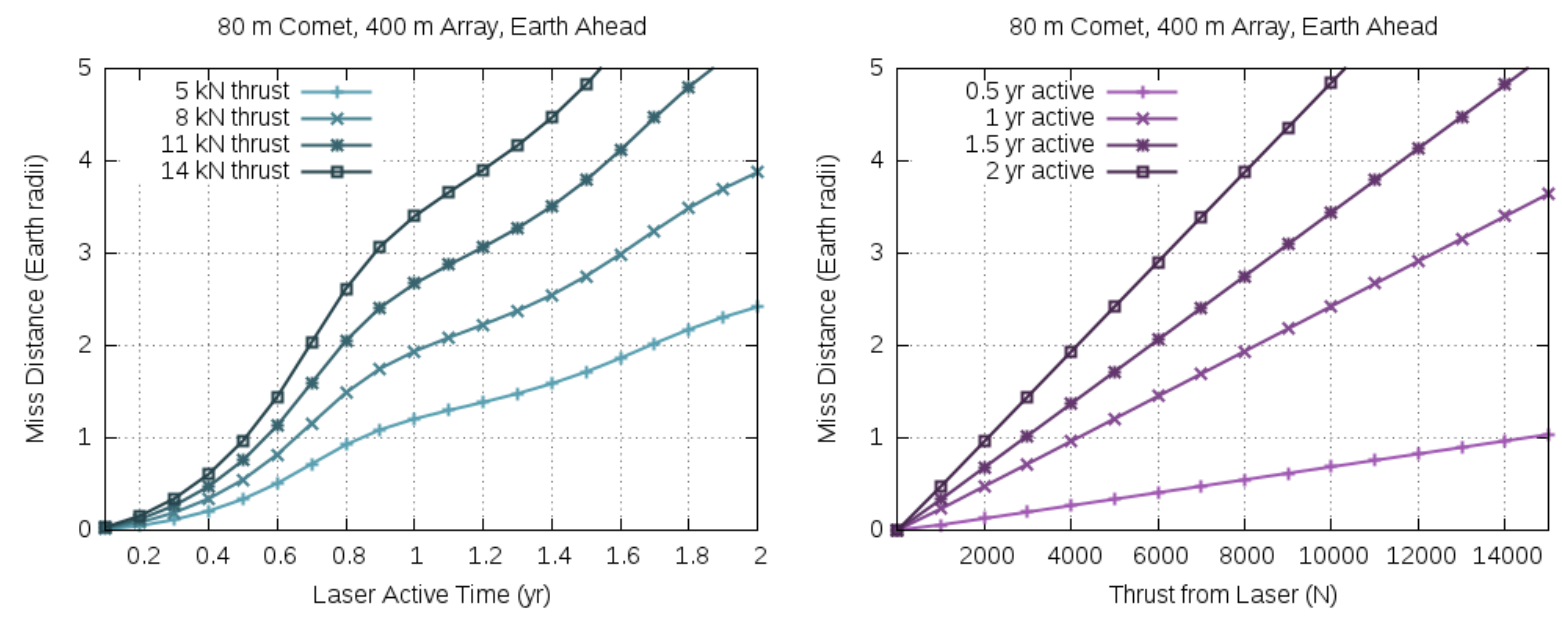

Figure 9. Deflection of an $80 \mathrm{~m}$ comet by a $400 \mathrm{~m}$ stand-off laser array: At 50\% solar-to-laser efficiency, the array produces a combined beam of $110 \mathrm{MW}$ optical power providing roughly $11 \mathrm{kN}$ thrust assuming $100 \mu \mathrm{N} / \mathrm{W}$. Miss distance increases quadratically with increasing laser active time to a certain point. Starting the laser even earlier results in a period where the laser spot diameter $D_{s}$ is bigger than the diameter of the comet's nucleus $D$ which still increases deflection (unlike the asteroid case which flattens out after the spot size exceeds $D_{c r i t}$ ) but increases less than quadratically (left). For a fixed laser array size, increasing efficiency and therefore thrust increases the miss distance of the comet linearly (right).

Below $~ 350 \mathrm{~m}$, a stand-off system becomes unable to deflect a comet or asteroid of any size. These smaller arrays may still be useful in mitigating very small threats $(\sim 20 \mathrm{~m})$ by vaporization or otherwise total disintegration. Structural analysis, however, is beyond the scope of these simulations which assume a target of constant mass.

In general, a stand-off system is significantly less effective than a similarly-sized stand-on mission. Due to the divergence of the laser beam over large distances by diffraction effects, a very large laser array of at least $1 \mathrm{~km}$ is needed to concentrate enough flux into a spot to ablate material off an asteroid. The lack of a transit time, however, makes a stand-off setup the only directed energy option for deflecting incoming asteroids on short notice. Furthermore, such stand-off systems are significantly more effective on long-period comets. These targets may approach in a trajectory unreachable by modern spacecraft propulsion systems making such a system stand out as one of the very few options available to mitigate such threats. With either case, a stand-off system needs a large array of at least several hundred meters is to be effective at deflecting any target and thus remains a long-term option rather than an immediate solution.

\section{CONCLUSIONS}

Directed energy is a promising technology for planetary defense. A modest stand-on DE-STARLITE mission of just $1 \mathrm{MW}(100 \mathrm{~N})$, which fits within an SLS Block 1 launch configuration, can deflect all known threats up to $500 \mathrm{~m}$ in diameter with 5 years of laser activity. That same system could deflect Tunguska or Chelyabinsk-sized asteroids in well under a year after arrival at the asteroid. With the strong dependence of deflection on laser active time, a much smaller and less expensive system could be equally effective given a decade or more of activity. On the other hand, stand-on systems are largely ineffective for deflecting targets on short notice due to the time required for transit to the asteroid.

In the absence of more than a few weeks of warning, the only option becomes a very large stand-off DE-STAR system. In addition to providing a last line of defense against threats which have evaded detection until shortly before impact, such a system may also provide one of the few options for defense against long period comets to which modern technology is generally incapable of reaching. A system of sufficient scale, however, may require decades to construct and so becomes a possibility only in the more distant future. 
The actual effectiveness of a deflection mission depends strongly on the target to be deflected. A mission optimized for one asteroid may be comparatively ineffective when applied to another, even of the same size. Orbital simulations provide a means for determining the specific mission requirements for targeting each specific threat. Planning, however, must begin long before an actual threat is identified. With orbital simulations, classes of threats can be identified and planned for ahead of time, minimizing the build out time thus maximizing the effectiveness of the system once a real threat is confirmed.

\section{ACKNOWLEDGEMENTS}

We gratefully acknowledge funding from the NASA California Space Grant NASA NNX10AT93H.

\section{REFERENCES}

[1] Belton, M.J.S., Morgan, T.H., Samarasinha, N.H. and Yeomans, D.K., eds. Mitigation of hazardous comets and asteroids. Cambridge University Press, 2004.

[2] Gritzner, C, and Kahle, R. "Mitigation technologies and their requirements." In Mitigation of Hazardous Comets and Asteroids, vol. 1, p. 167. Cambridge Univ. Press, Cambridge (2004).

[3] Colombo, C, Vasile, M. and Radice, G. "Semi-analytical solution for the optimal low-thrust deflection of near-earth objects." Journal of Guidance, Control, and Dynamics 32.3 (2009): 796-809.

[4] Cuartielles, J.P.S., et al. "A Multi-criteria Assessment of Deflection Methods for Dangerous NEOs." AIP conference proceedings. Vol. 886. 2007.

[5] Morrison, D, Harris, A.W., Sommer, G., Chapman, C.R. and Carusi, A. "Dealing with the impact hazard." Asteroids III"(ed. W. Bottke et al., Univ. Ariz. Press) (2002): 739-754.

[6] Koenig, J.D., and Chyba, C.F. "Impact deflection of potentially hazardous asteroids using current launch vehicles." Science and Global Security 15, no. 1 (2007): 57-83.

[7] Melosh, H. J., and E. V. Ryan. "Asteroids: Shattered but not dispersed." Icarus 129, no. 2 (1997): 562-564.

[8] McInnes, C.R. "Deflection of near-Earth asteroids by kinetic energy impacts from retrograde orbits." Planetary and Space Science 52.7 (2004): 587.

[9] Conway, B.A. "Optimal interception and deflection of Earth-approaching asteroids using low-thrust electric propulsion." Mitigation of hazardous comets and asteroids Belton, M.J.S., Morgan T.H., Samarasinha, N, and Yeomans, D.K. (2004): 292-312.

[10] Hyland, D. C., H. A. Altwaijry, S. Ge, R. Margulieux, J. Doyle, J. Sandberg, B. Young, X. Bai, J. Lopez, and N. Satak. "A permanently-acting NEA damage mitigation technique via the Yarkovsky effect." Cosmic Research 48, no. 5 (2010): 430-436.

[11] Vasile, M., and Maddock, C.A. "On the deflection of asteroids with mirrors." Celestial Mechanics and Dynamical Astronomy 107, no. 1 (2010): 265-284.

[12] Wie, B. "Hovering control of a solar sail gravity tractor spacecraft for asteroid deflection." In Proceedings of the 17th AAS/AIAA Space Flight Mechanics Meeting, AAS, vol. 7, p. 145. 2007.

[13] Walker, R., Izzo, D., de Negueruela, C., Summerer, L., Ayre, M., and Vasile, M. "Concepts for Near-Earth Asteroid deflection using spacecraft with advanced nuclear and solar electric propulsion systems." Journal of the British Interplanetary Society 58, no. 7-8 (2005): 268-278.

[14] Schweickart, R., Chapman, C., Durda, D., and Hut, P. "Threat mitigation: the gravity tractor." arXiv preprint physics/0608157 (2006).

[15] Lu, E.T., and Love, S.G. "A gravitational tractor for towing asteroids." arXiv preprint astro-ph/0509595 (2005).

[16] Olds, J., Charania, A., \& Schaffer, M. G. (2007, March). Multiple mass drivers as an option for asteroid deflection missions. In 2007 Planetary Defense Conference, Washington, DC, Paper (pp. S3-7).

[17] Gibbings, M. A., Hopkins, J. M., Burns, D., \& Vasile, M. (2011). On Testing Laser Ablation Processes for Asteroid Deflection, 2011 IAA Planetary Defense Conference, Bucharest, Romania. 
[18] Maddock, C, Cuartielles, J.P.S., Vasile, M. and Radice, G. "Comparison of Single and Multi-Spacecraft Configurations for NEA Deflection by Solar Sublimation." In AIP Conference Proceedings, vol. 886, p. 303. 2007.

[19] Kahle, R., Hahn, G., and Kührt, E. "Optimal deflection of NEOs en route of collision with the Earth." Icarus 182, no. 2 (2006): 482-488.

[20] Vasile, M., \& Maddock, C. A. (2010). On the deflection of asteroids with mirrors. Celestial Mechanics and Dynamical Astronomy, 107(1-2), 265-284.

[21] Kosmo, K., Pryor, M., Lubin, P., Hughes, G.B., O’Neill, H., Meinhold, P., Suen, J., C., Riley, J., Griswold, J., Cook, B.V., Johansson, I.E., Zhang, Q., Walsh, K., Melis, C., Kangas, M., Bible, J., Motta, Brashears, T., Mathew, S. and Bollag, J. "DE-STARLITE - A Directed Energy Planetary Defense Mission,” Nanophotonics and Macrophotonics for Space Environments VIII, edited by Edward W. Taylor, David A. Cardimona, Proc. of SPIE Vol. 9226 (Aug, 2014).

[22] Lubin, P., Hughes, G.B., Bible, J., Bublitz, J., Arriola, J., Motta, C., Suen, J., Johansson, I., Riley, J., Sarvian, N., Clayton-Warwick, D., Wu, J., Milich, A., Oleson, M., Pryor, M., Krogen, P., Kangas, M., and O’Neill, H. “Toward Directed Energy Planetary Defense,” Optical Engineering, Vol. 53, No. 2, pp 025103-1 to 025103-18 (Feb 2014), doi: 10.1117/1.OE.53.2.025103.

[23] Thiry, N. and Vasile, M. "Recent Advances in Laser Ablation Modelling for Asteroid Deflection Methods," Nanophotonics and Macrophotonics for Space Environments VIII, edited by Edward W. Taylor, David A. Cardimona, Proc. of SPIE Vol. 9226 (Aug, 2014).

[24] Ahrens T.J. and A.W. Harris. 1992. "Deflection and fragmentation of near-Earth asteroids.” Nature 360(6403): 429433.

[25] Johansson, I.E., Tsareva, T., Griswold, J., Lubin, P., Hughes, G.B., O’Neill, H., Meinhold, P., Suen, J., Zhang, Q., J., Riley, J. Walsh, K., Brashears, T., Bollag, J., Mathew, S. and Bible, J. "Effects of asteroid rotation on directed energy deflection,” Nanophotonics and Macrophotonics for Space Environments VIII, edited by Edward W. Taylor, David A. Cardimona, Proc. of SPIE Vol. 9226 (Aug, 2014).

[26] Hughes, G.B., Lubin, P., Griswold, J., Bozinni, D., O’Neill, H., Meinhold, P., Suen, J., Bible, J., Riley, J., Johansson, I.E., Pryor, M. and Kangas, M. "Optical modeling for a laser phased-array directed energy system (Invited Paper)," Nanophotonics and Macrophotonics for Space Environments VIII, edited by Edward W. Taylor, David A. Cardimona, Proc. of SPIE Vol. 9226 (Aug, 2014).

[27] Lubin, Philip, Gary B. Hughes, Johanna Bible, Jesse Bublitz, Josh Arriola, Caio Motta, Jon Suen, Isabella Johansson, Jane Wu, Andrew Milich , Mitch Oleson and Mark Pryor. "DE-STAR: A Planetary Defense and Exploration System.” Proc. Of SPIE, Optics \& Photonics (2013).

[28] Hughes, G. B., Lubin, P. M., Bible, J., Bublitz, J., Arriola, J., Motta, C., Suen, J., Johannson, I., Riley, J., Sarvian, N., Wu, J., Milich, A., and Pryor, M., "DE-STAR: Phased Array Laser Technology for Planetary Defense and Other Scientific Purposes," Proc. of SPIE, Optics \& Photonics (2013).

[29] Lubin, P. M., Hughes, G. B., Bible, J. and Johannson, I. "Directed Energy for Planetary Defense and Exploration Applications to Relativistic Propulsion and Interstellar and Intergalactic Communications", Journal of the British Interplanetary Society - JBIS, 2015

[30] Duncan, M.J., Levison, H.F., and Lee, M.H. “A Multiple Time Step Symplectic Algorithm for Integrating Close Encounters”, Astronomical Journal 116, (1998) 2067. 